\title{
Synthesis and Characterization of NLO Material L-Valine L- Valinium Perchlorate Monohydrate for Photonics Applications
}

\author{
Vasudevan Paranthaman ${ }^{* 1}$ and Jeyaraman Devaraj ${ }^{2}$ \\ ${ }^{I}$ Department of Physics, Rajalakshmi Engineering College, Thandalam, Chennai-602105, India. \\ ${ }^{2}$ Department of Physics, Presidency College, Chennai-600 005, India.
}

Received May 11, 2020; accepted September 19, 2020; published September 30, 2020

\begin{abstract}
L-valine L-valinium perchlorate monohydrate single crystal has been synthesized by slow evaporation technique at room temperature. The crystal structure and space group of the crystal were confirmed by a single crystal X-ray diffractometer. Optical behavior of the crystal was analyzed by using a UV-visible spectrophotometer. Thermal stability was discussed by using a thermo gravimetric analysis. Mechanical strength of the grown crystal was studied using a Vickers microhardness tester. The nonlinear optical property was explored to confirm the second harmonic generation efficiency of the grown crystal. These preliminary investigations suggest that the title compound can serve as a potential material for photonics applications.
\end{abstract}

The search for non-linear optical (NLO) materials have attracted considerable interest among researchers over the past two decades for their potential applications in optical communications, laser technology, optical storage devices and optoelectronics [1-3]. In this respect, amino acids are intriguing materials for NLO applications as they have proton donor carboxyl acid groups $\left(\mathrm{COO}^{-}\right)$and proton acceptor amino groups $\left(\mathrm{NH}_{2}^{-}\right)$. In recent years, attention has been focused on amino acid with organic and inorganic complex crystals, in order to combine highoptical nonlinearties associated with an organic acid having favourable mechanical and thermal properties of an inorganic acid which makes the materials suitable for second harmonic generation. Hence, some of the amino acids such as L-arginine, L-alanine, L-phenyalanine, Lhistidine and L-threonine have been subjected to formation of single crystals with various organic and inorganic acids. As literature survey reports, semiorganic single crystals like L-arginine hydrobromide monohydrate, L-alanine alaninium nitrate, L-histidine dihydrochloride, etc., [4-5] are some of the examples which proved suitable for photonics applications. Therefore, we have chosen L-valine and perchloric acid as starting materials to form semi-organic nonlinear optical single crystal by a slow evaporation technique at room temperature for the investigations. Valine is a branched chain of $\alpha$-amino acid having an aliphatic nonpolar side chain. Several new complexes incorporating L-valine have been recently crystallized and their structural, optical, electrical, mechanical and thermal properties have been

*E-mail: vasudevan.p@rajalakshmi.edu.in investigated like glycyl L-valine, L-valine cadmium chloride monohydrate, L-valine zinc hydrochloride, Lvaline cadmium bromide, etc., [6-7]. However, the structure of L-valine L-valinium perchlorate monohydrate (LVLVPM) single crystal has been reported earlier [8]. The other properties such as optical, thermal and mechanical properties of the single crystal are seldom found in literature. Therefore, in the present investigation, single crystals of LVLVPM have been synthesized and characterized by single crystal XRD, UV-visible spectral analysis, thermo gravimetric analysis (TGA), Vickers microhardness and nonlinear optical (NLO) test to examine the potential material for device fabrication.

Analytical grade of L-valine and perchloric acid along with doubled distilled water were used for preparation of an LVLVPM single crystal. A solution was prepared by dissolving L-valine and perchloric acid in water with a 2:1 stoichiometricratio molar ratio and stirred well using a magnetic stirrer to get a homogeneous solution. The solution was then allowed for slow evaporation at room temperature. As a result, the solution gradually reached supersaturation leading to a nucleation phenomenon followed by the growth mechanism. Needle shaped and colourless crystals of LVLVPM with the size $35 \mathrm{~mm} \times 5 \mathrm{~mm} \times 4 \mathrm{~mm}$ have been formed during the period of 30 days with more transparency. The product was then purified by repeated recrystallization process. Fig. 1 shows as-grown LVLVPM single crystal. The reaction for the formation of the crystal is shown below:

$\mathrm{C}_{5} \mathrm{H}_{11} \mathrm{NO}_{2} \cdot \mathrm{C}_{5} \mathrm{H}_{11} \mathrm{NO}_{2}+\mathrm{HClO}_{4} \rightarrow \mathrm{C}_{5} \mathrm{H}_{12} \mathrm{NO}_{2}{ }^{+} \cdot \mathrm{ClO}_{4}{ }^{-} \cdot \mathrm{C}_{5} \mathrm{H}_{11} \mathrm{NO}_{2} \cdot \mathrm{H}_{2} \mathrm{O}$

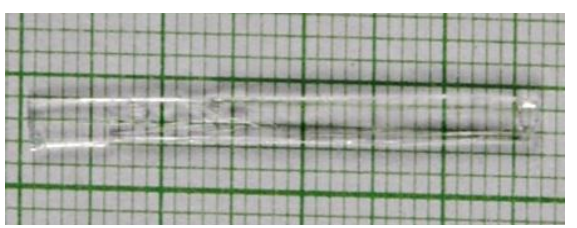

Fig. 1. As-grown LVLVPM single crystal.

Single crystal XRD studies were carried out using a Bruker Kappa APE XII single X-ray diffractometer to determine the lattice parameters and space group of the grown crystal. Optical absorption measurements were 
made using a Perkin Elmer Lamda 35 UV-VIS-NIR spectrophotometer with a resolution of $2 \mathrm{~nm}$ in the region from $200 \mathrm{~nm}$ to $1200 \mathrm{~nm}$ to measure the absorption range of the crystal. The TGA studies of the sample were carried out using the instrument STA 449 F1\&F3 Jupiter series in a temperature range of $30^{\circ} \mathrm{C}$ to $500^{\circ} \mathrm{C}$ in nitrogen atmosphere. Microhardness measurements of the grown crystal were carried out using a Reichert MD 4000E Ultra microhardness tester with a diamond pyramid indenter attached to an optical microscope. The nonlinear optical property of the crystal was confirmed by the Kurtz and Perry powder technique using a Q-switched high energy Nd: YAG laser (QUANTA RAY model LAB-170-10).

In order to identify the title compound and its lattice parameters, a single X-ray diffraction analysis was used. The experimental results reveal that the LVLVPM has been crystallized in a monoclinic structure with space group $\mathrm{P} 2{ }_{1}$. Hence the crystal has been classified as noncentrosymmetric group. Unit cell parameters are $a=$ $11.227 \AA, b=5.82 \AA, c=13.08 \AA, \alpha=\gamma=90^{\circ}$ and $\beta=91^{\circ}$. The volume of the system is $V=856.67 \AA^{3}$. These results are found to be in good agreement with the recently reported literature value [9]. The non-centrosymmetric nature of the grown crystal fulfills the important criterion for exhibiting NLO property.

The recorded absorption spectrum is shown in Fig. 2. From the observed spectrum, the lower cut-off wavelength is found to be $251 \mathrm{~nm}$ and there are no prominent absorption peaks found in the visible region. However, the minor peaks found in the visible region are due to phonon absorption, which indicates the absence of overtones. An absorption peak obtained at $222 \mathrm{~nm}$ shows $\mathrm{n}$ to $\pi^{*}$ transitions of the carbonyl group [10-12].

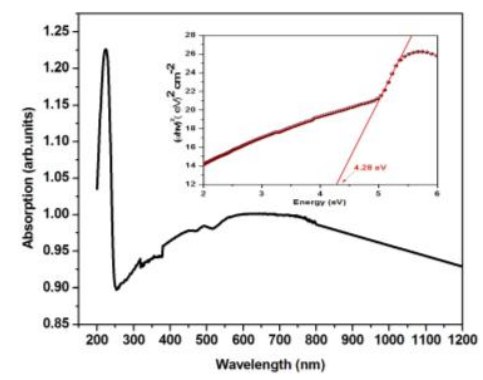

Fig. 2. UV-vis-NIR absorption spectrum and band gap of LVLVPM crystal.

The absence of absorption in the near infrared region provides access to the laser wavelength of $1064 \mathrm{~nm}$ from Nd: YAG source for second harmonic generation. Hence, UV absorption studies reveal that the grown LVLVPM crystal is one of the suitable materials for exhibiting second harmonic generation in the entire visible and near infrared region. The Tauc's plot of $(\alpha h v)^{2}$ against the photon energy $(\mathrm{h} v)$ at room temperature (Inset of Fig. 2) shows a linear behavior, ( $\alpha$-absorption coefficient and hPlanck constant) which can be considered as evidence for indirect transition of the process [13]. Using Tauc's plot, the band gap of the LVLVPM crystal was found to be $4.28 \mathrm{eV}$. The higher band gap value shows that the defect inclusion in the grown crystal is very low and the material can also exhibit dielectric behavior.

Thermal analysis was performed on the grown crystal to study thermal stability. The thermo gravimetric analysis of the grown crystal LVLVPM was carried out between the temperature $20^{\circ} \mathrm{C}$ and $500^{\circ} \mathrm{C}$ at a heating rate of $20^{\circ} \mathrm{C} / \mathrm{min}$ under nitrogen atmosphere. The recorded thermogram is shown in Fig. 3. From the thermogram plot, there is a single stage weight loss starting at $235^{\circ} \mathrm{C}$ but in the range between 20 and $235^{\circ} \mathrm{C}$ no weight loss occurred. It indicates the absence of physically absorbed water molecules in the sample. Therefore, the title compound is thermally stable up to $235^{\circ} \mathrm{C}$. The total weight loss is nearly completed at $90 \%$ and the resulting residue is at $10 \%$ till $500^{\circ} \mathrm{C}$. Hence, TG analysis reveals that LVLVPM crystal is suitable for photonic device fabrication up to $235^{\circ} \mathrm{C}$ without any thermal damage.

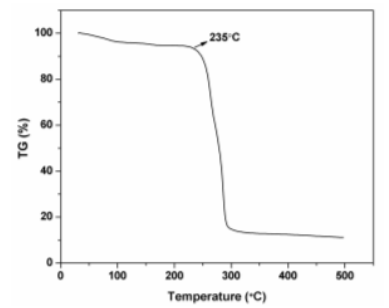

Fig. 3. TGA curve of LVLVPM crystal.

Hardness is a significant mechanical property to be studied for the device fabrication in the field of optoelectronics. Microhardness study of the grown crystal was carried out using a Reichert MD 4000E Ultra microhardness tester with a diamond pyramid indenter attached to an optical microscope. To get accurate results, a well polished crystal was selected arbitrarily to study the mechanical strength of the crystal. The applied load $P$ varied from $5 \mathrm{~g}$ to $30 \mathrm{~g}$ and the corresponding time of indentation was kept constant as $15 \mathrm{~s}$ for all trials. The diagonal lengths of indentation were determined for various loads $(\mathrm{P})$ in $\mathrm{g}$. The Vickers microhardness number $\left(H_{v}\right)$ was calculated [14] using the relation:

$$
H_{\mathrm{v}}=1.8544 P / d^{2} \mathrm{~kg} / \mathrm{mm}^{2},
$$

where $\mathrm{P}$ is the applied load (expressed in $\mathrm{kg}$ ) and $\mathrm{d}$ is the mean diagonal length (expressed in $\mathrm{mm}$ ) of the indenter impression. The variation of $H_{v}$ with applied load $P$ is shown in Fig. 4. The plot indicates that hardness increases with applied load up to $30 \mathrm{~g}$ and with a further increase in load, cracks start to appear on the surface of crystal. From 
the result, it is concluded that an increase in microhardness with an increase in load is in good agreement with the reverse indentation size effect (RISE). This might be explained based on the depth of penetration by an indenter. When the load increases, initially the surface layers are penetrated and on further load increase the inner surface layer of the crystal is penetrated by the indenter. Hence, the increase of hardness number is due to the overall effect on the surface and inner layers of the grown crystal. The increasing tendency of hardness with a load up to $30 \mathrm{~g}$ helps understand Meyer's law and Onistch condition. The established Meyer's law gives the relation between load $P$ and size $d$ of the indentation as:

$$
P=\mathrm{A} d^{n}
$$

where $n$ is Meyer's number and A is the constant. By plotting $\log P$ vs $\log d$ (Inset of Fig. 4), the work hardening index value of LVLVPM was found to be 2.18 . According to Onistch, if the $\mathrm{n}$ value lies between 1.0 and 1.6 , the material is hard and the $n$ value is above 1.6 , the material is soft. Hence, it is concluded that the grown crystal material is of a soft category. Therefore, the material LVLVPM crystal is capable of sustaining the load up to $30 \mathrm{~g}$ without any defect during the fabrication of optoelectronic devices.

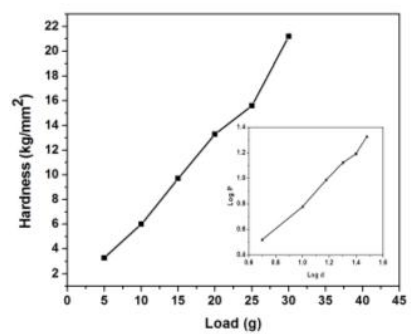

Fig. 4. Vickers microhardness of LVLVPM crystal.

The grown crystal LVLVPM was powdered and well packed in a capillary tube. A high intense beam of laser wavelength $1064 \mathrm{~nm}$ was allowed to illuminate the sample with a pulse width of $8 \mathrm{~ns}$ and a repetition rate of $10 \mathrm{~Hz}$. The emission of green radiation $532 \mathrm{~nm}$ from the sample confirms that the material exhibits a nonlinear optical property. The second harmonic generation efficiency of the title compound was found to be 1.5 times that of a standard material KDP [15]. The measurements were made for some other amino acid derivatives, too. The results reveal that LVLVPM crystal is found to possess maximum SHG efficiency when compared to that of Lphenylalanium nitrate $(0.26)$, L-phenylalanium maleate (0.27), L-alanine (0.33) and triglycine zinc chloride $(0.5)$ [16]. Moreover, the grown crystal has the advantage of combining thermal and mechanical robustness with high nonlinear behavior required for photonic applications.

In conclusion, L-valine L-valinium perchlorate monohydrate single crystal was successfully grown by a slow evaporation technique at room temperature. The single crystal XRD study reveals that crystal belongs to monoclinic structure and non-centrosymmetric space group $\mathrm{P} 2{ }_{1}$. The optical absorption spectral analysis reveals that the crystal is transparent in the entire UV-vis-NIR region with the lower cut-off wavelength as $251 \mathrm{~nm}$ and the band gap was found to be $4.28 \mathrm{eV}$. Thermal behavior of the grown material has been analyzed using thermal studies. Vickers microhardness reveals the LVLVPM belongs to a soft material category. The Kurtz and Perry powder technique confirms that LVLVPM is one of the promising nonlinear optical materials and the SHG efficiency of the grown crystal is 1.5 times that of standard material KDP and the result is compared with some other amino acid derivative crystals. The above studies depict that material is useful for nonlinear optical applications.

\section{References}

[1] D.J. Williams, Nonlinear Optical Properties of Organic and Polymer Materials (ACS Symposium series 233, American Chemical Society, Washington, DC, 1983).

[2] K. Bouchouit, Z. Sofiani, B. Derkowska, S. Abed, N. Benalicherif, M. Bakasse, B. Sahraoui, Opt. Commun. 278, 180 (2007), https://doi.org/10.1016/j.optcom.2007.05.068

[3] K. Bouchouit, H. Bougharraf, B. Derkowska-Zielinska, N. BenaliCherif, B. Sahraoui, Opt. Mater. 48, 215 (2015), https://doi.org/10.1016/j.optmat.2015.07.035

[4] J.H. Joshi, S. Kalainathan, M.J. Joshi, K.D. Parikh, Arab. J. Chem. 13, 5018 (2020), https://doi.org/10.1016/j.arabjc.2020.01.024

[5] A. Vijayakumar, A. Ponnuvel, A. Sasikala, Mater. Today 14, 338 (2019), https://doi.org/10.1016/j.matpr.2019.04.156

[6] C. Usha, R. Sathakuamri, L.J.D. Sajan, R.Meenakshi, A. Sinthiya, Heliyon 5, e01574 (2019), https://doi.org/10.1016/j.heliyon.2019.e01574

[7] P. Maadeshwaran, J. Chandrasekaran, Optik 122, 1128 (2011), https://doi.org/10.1016/j.ijleo.2010.07.006

[8] S. Pandiyaran, M. Umadevi, R.K. Rajaraman, V.K. Ramakrishnan, Spectrochim. Act A Mol. 62, 630 (2005), https://doi.org/10.1016/j.saa.2005.02.008

[9] S. Pandiarajan, B. Sridhar, R.K. Rajaram, Acta Crystallogr. E, 57, 0466 (2001), https://doi.org/10.1107/S160053680100681X

[10] M. Lydia Caroline, S. Vasudevan, Mater. Lett. 63, 41 (2009), 10.1016/j.matlet.2008.08.059

[11] J. Tauc, R. Grigorovici, A. Vancu, Phy. Solid. Stat. 15, 627 (1966), https://doi.org/10.1002/pssb.19660150224

[12] J. Tauc, A. Menth, D.L. Wood, Phys. Rev. Lett. 25, 749 (1970), https://doi.org/10.1103/PhysRevLett.25.749

[13] B. Thirumalaiselvam, R. Kanagadurai, D. Jayaraman, V. Natarajan, Opt.Mater. 37, 74 (2014), https://doi.org/10.1016/j.optmat.2014.05.002

[14] J. Bowman, M. Bevis, Colloid Polym. Sci. 255, 954 (1977), https://link.springer.com/article/10.1007/BF01776201

[15] S.K. Kurtz, T.T. Perry, J. Appl. Phy. 39, 3798 (1968), https://doi.org/10.1063/1.1656857

[16] M. Prakash, D. Geetha, M. Lydia Caroline, Spectrochim. Act. A Mol. 81, 48 (2011), https://doi.org/10.1016/j.saa.2011.05.039 